\title{
SI: DNA Origami Chromophore Scaffold Exploiting HomoFRET Energy Transport to Create Molecular Photonic Wires
}

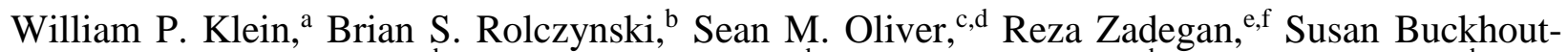
White, ${ }^{\mathrm{a}}$ Mario G. Ancona, ${ }^{\mathrm{b}}$ Paul D. Cunningham, ${ }^{\mathrm{b}}$ Joseph S. Melinger, ${ }^{\mathrm{b}}$ Patrick M. Vora, ${ }^{\mathrm{c}, \mathrm{d}}$ Wan Kuang, ${ }^{\mathrm{g}}$ Igor L. Medintz, ${ }^{\mathrm{a},{ }^{*}}$ and Sebastián A. Díaz, ${ }^{\mathrm{a}, *}$

${ }^{a}$ Center for Bio/Molecular Science and Engineering, Code 6900, U.S. Naval Research Laboratory, Washington, DC 20375 USA

bElectronic Science and Technology Division, Code 6800, U.S. Naval Research Laboratory, Washington, DC 20375, USA

${ }^{c}$ Department of Physics and Astronomy, George Mason University, Fairfax, VA 22030, USA

${ }^{d}$ Quantum Materials Center, George Mason University, Fairfax, VA 22030, USA

${ }^{\mathrm{e}}$ Micron School of Materials Science and Engineering and the Computer Science Department, Boise State University, Boise, ID 83706, USA

fDepartment of Nanoengineering, The Joint School of Nanoscience and Nanoengineering, North Carolina A\&T State University, Greensboro, NC 27401

'Department of Electrical and Computer Engineering, Boise State University, Boise, ID 83706, USA

*Corresponding Author Email: igor.medintz@,nrl.navy.mil ; sebastian.diaz@nrl.navy.mil

\section{DNA Sequence Tables}

Table S1- Unlabeled strands for six-helix bundle nanotube.

\begin{tabular}{|l|l|c|c|}
\hline Name & Sequence 5->3 & Length & Mp. $\left({ }^{\circ} \mathbf{C}^{\mathbf{1}, \mathbf{2}}\right.$ \\
\hline Staple 1 & TACAAAGGCTAGAGCTCGAATT & 22 & 58.4 \\
\hline Staple 2 & ACAGGAAGATTGTATTTAAATTGTAAACGTTAATATT & 37 & 65.8 \\
\hline Staple 3 & TCTAGAGGATCCCCGGGTACCTCAGGTCATTGCCT & 5 & 78.8 \\
\hline Staple 4 & TTGTTAAAGTCGGATTCTCCCGGTTGATAATCAG & 4 & 71.6 \\
\hline Staple 5 & TGGGAACAAACGGCGGATTGA & 21 & 61.2 \\
\hline Staple 6 & CGTAATGGGATAGGTTACGTGAACGGTAATCGT & 33 & 72.5 \\
\hline Staple 7 & TGGTGTAGCTGCAGGTCGAC & 20 & 62.5 \\
\hline Staple 8 & TGCATGCATGGGCGCATCGTA & 21 & 63.2 \\
\hline Staple 9 & TTTGTTAAATTTTAACCAATAGGGCAACTGTTGGG & 35 & 69.4 \\
\hline Staple 10 & ACAACCCATTCGCGTTAAATT & 21 & 55.4 \\
\hline Staple 11 & AACATTAAATGTGAGCGAGTA & 21 & 53.4 \\
\hline Staple 12 & ACCGTGCATCTGCCTTTCATC & 21 & 62.2 \\
\hline Staple 13 & CAAAAATAATTCGGCCATTCGCCATTCAGGCTGCAACGCCAT & 42 & 79.1 \\
\hline Staple 14 & CTTCCTGTAGCCAGCAGTTTG & 21 & 61.2 \\
\hline Staple 15 & AGGGGACGACGACAGCTTTCCGGCACCGCTTCTGGT & 36 & 82.4 \\
\hline Staple 16 & CCTCAGGAGCCAGTGTTGATCAGCATGTTGTAA & 33 & 73.7 \\
\hline Staple 17 & AACGACGAGATCGCACTCCAGCCCGTATCGG & 31 & 77.5 \\
\hline Staple 18 & GCCGGGGGGGATGTGCTGCAAAACTAGCATGTCAATCATA & 40 & 80.1 \\
\hline Staple 19 & AAGGGCGATCGGTGCGGGCAAAAGCCCCAAAA & 32 & 77.5 \\
\hline Staple 20 & CTCTTCGCTATTACGCCAGCTTGTACCC & 28 & 71.8 \\
\hline Staple 21 & GGCGAAAAAACCAGGCAAAGCCGTCTGGC & 29 & 74.8 \\
\hline Staple 22 & AAGGCGATTAAGTTGGGTAACGAATCGA & 28 & 67.2 \\
\hline Staple 23 & GCCAGGGTTTTCCCAGTCACGGAGAGTCTGGAGCAAACAAGA & 42 & 81.9 \\
\hline
\end{tabular}

1 Calculated melting point (Mp) of ssDNA strands. Oligonucleotide Properties Calculator (https://www.idtdna.com/). ${ }^{2}$ The salt adjusted melting temperature was used for all Mp calculations. 
Table S2- Labeled strands for six-helix bundle nanotube.

\begin{tabular}{|l|l|c|c|}
\hline Name & Sequence 5->3 & Length & Mp. $\left({ }^{\circ} \mathbf{C}\right)^{\mathbf{1 , 2}}$ \\
\hline Staple 2 (AF647) & 647-TCTAGAGGATCCCCGGGTACCTCAGGTCATTGCCT & 35 & 69.1 \\
\hline Staple 4 (AF488) & AF488-TTGTTAAAGTCGGATTCTCCCGGTTGATAATCAG & 34 & 71.6 \\
\hline Staple 5 Cy3.5) & Cy3.5-TGGGAACAAACGGCGGATTGA & 21 & 61.2 \\
\hline Staple 6 Cy3.5) & Cy3.5-CGTAATGGGATAGGTTACGTGAACGGTAATCGT & 33 & 72.5 \\
\hline Staple 7 Cy3.5) & Cy3.5-TGGTGTAGCTGCAGGTCGAC & 20 & 62.5 \\
\hline Staple 8 Cy3.5) & 647-TGCATGCATGGGCGCATCGTA & 21 & 63.2 \\
\hline Staple 9 (AF488) & AF488-TTTGTTAAATTTTAACCAATAGGGCAACTGTTGGG & 35 & 69.4 \\
\hline Staple 10 (Cy3.5) & Cy3.5-ACAACCCATTCGCGTTAAATT & 21 & 55.4 \\
\hline Staple 11 (Cy3.5) & Cy3.5-AACATTAAATGTGAGCGAGTA & 21 & 53.4 \\
\hline Staple 12 (Cy3.5) & Cy3.5-ACCGTGCATCTGCCTTTCATC & 21 & 61.2 \\
\hline Staple 13 (Cy3.5) & Cy3.5- & 42 & 71.9 \\
\hline Staple 14 (Cy3.5) & Cy3.5-CTTCCTGTAGCCAGCAGTTTG & 21 & 61.2 \\
\hline Staple 15 (Cy3.5) & Cy3.5-AGGGGACGACGACAGCTTTCCGGCACCGCTTCTGGT & 36 & 82.4 \\
\hline Staple 16 (Cy3.5) & Cy3.5-CCTCAGGAGCCAGTGTTGATCAGCATGTTGTAA & 33 & 73.7 \\
\hline
\end{tabular}

1 Calculated melting point (Mp) of ssDNA strands. Oligonucleotide Properties Calculator (https://www.idtdna.com/). ${ }^{2}$ The salt adjusted melting temperature was used for all Mp calculations.

\section{Preparation and Purification Protocol for Cut M13}

The scaffold for the DNA origami wire was prepared by cleaving commercially purchased M13mp18 plasmid (Bayou Biolabs) with two restriction enzymes, EcoRI and BglII, (New England Biolabs) to create a 704 nucleotide long scaffold. To create the double-stranded region on M13mp18 plasmid for the restriction enzymes another two 36 basepair ssDNA oligos were required:

\section{EcoR1 Seq GCT CGA ATT CGT AAT CAT GGT CAT AGC TGT TTC CTG}

\section{BglII Seq CCG GAG AGG GTA GCT ATT TTT GAG AGA TCT ACA AAG}

The salt adjusted melting temperature for these strands was $74.2{ }^{\circ} \mathrm{C} .64 \mu \mathrm{L}(10 \mu \mathrm{M})$ of the two complementary 36 basepair ssDNA oligos were added to $800 \mu \mathrm{L}$ stock M13mp18 (concentration $360 \mathrm{nM}$ ). 18.6 $\mu \mathrm{L} \mathrm{MgCl}_{2}(1000 \mu \mathrm{M})$ was added to increase the affinity for binding. The primer strands were added to the M13 in a two to one excess primers to M13 ratio. The solution was then split into $200 \mu \mathrm{L}$ Lo Bind centrifuge tubes, $100 \mu \mathrm{L}$ of solution in each was then annealed using the following program: $90{ }^{\circ} \mathrm{C} 1$ minute, $90{ }^{\circ} \mathrm{C}-1.0{ }^{\circ} \mathrm{C}$ per 5 seconds $10 \mathrm{X}, 80{ }^{\circ} \mathrm{C}-1.0{ }^{\circ} \mathrm{C}$ per 15 seconds $15 \mathrm{X}, 65^{\circ} \mathrm{C}-1.0{ }^{\circ} \mathrm{C}$ per 30 seconds $45 \mathrm{X}$, hold $4{ }^{\circ} \mathrm{C}$. After the anneal process was complete the samples were combined in a $1.5 \mathrm{~mL}$ centrifuge tube. $95 \mu \mathrm{L}$ of $10 \times$ Fast Digest Buffer was added followed by $16 \mu \mathrm{L}$ of each enzyme. The sample was again split into $200 \mu \mathrm{L}$ Lo Bind centrifuge tubes and annealed at $37{ }^{\circ} \mathrm{C}$ for two hours and then $85{ }^{\circ} \mathrm{C}$ for 10 minutes 
to denature the enzymes. The short $704 \mathrm{bp}$ cut was separated from the long M13 ssDNA using 1.5\% agarose gel electrophoresis. The resulting gel was illuminated with UV light on a TLC plate. Two bands were observed and the lower band containing the M13 cut was extracted from the agarose gel using Freeze and Squeeze tubes. The gel containing the M13 cut was cut up using a razor blade and then placed in the tube, the tube was then spun at 17,000 ref for 3 minutes. In order to increase the extraction yield, after the first spin $100 \mu \mathrm{L}$ of $1 \times$ TAE was added to the gel and stirred. The tube was spun again three more times adding $100 \mu \mathrm{L}$ of $1 \times \mathrm{TAE}$ each time.

The properties for M13 cut are as follows: M13 Cut ssDNA Molecular Wt. $=216,917.5 \mathrm{~g} \mathrm{~mole}^{-1}, 1.0 \mathrm{ABS}$ $=135 \mathrm{nM}, 1 \mathrm{~mL}=29.3 \mathrm{mg}$.

\section{M13 Cut Sequence}

TCG AGC TCG GTA CCC GGG GAT CCT CTA GAG TCG ACC TGC AGG CAT GCA AGC TTG GCA CTG GCC GTC GTT TTA CAA CGT CGT GAC TGG GAA AAC CCT GGC GTT ACC CAA CTT AAT CGC CTT GCA GCA CAT CCC CCT TTC GCC AGC TGG CGT AAT AGC GAA GAG GCC CGC ACC GAT CGC CCT TCC CAA CAG TTG CGC AGC CTG AAT GGC GAA TGG CGC TTT GCC TGG TTT CCG GCA CCA GAA GCG GTG CCG GAA AGC TGG CTG GAG TGC GAT CTT CCT GAG GCC GAT ACG GTC GTC GTC CCC TCA AAC TGG CAG ATG CAC GGT TAC GAT GCG CCC ATC TAC ACC AAC GTA ACC TAT CCC ATT ACG GTC AAT CCG CCG TTT GTT CCC ACG GAG AAT CCG ACG GGT TGT TAC TCG CTC ACA TTT AAT GTT GAT GAA AGC TGG CTA CAG GAA GGC CAG ACG CGA ATT ATT TTT GAT GGC GTT CCT ATT GGT TAA AAA ATG AGC TGA TTT AAC AAA AAT TTA ACG CGA ATT TTA ACA AAA TAT TAA CGT TTA CAA TTT AAA TAT TTG CTT ATA CAA TCT TCC TGT TTT TGG GGC TTT TCT GAT TAT CAA CCG GGG TAC ATA TGA TTG ACA TGC TAG TTT TAC GAT TAC CGT TCA TCG ATT CTC TTG TTT GCT CCA GAC TCT CAG GCA ATG ACC TGA TAG CCT TTG TA

\section{Annealing and Purification of DNA origami}

The DNA origami was prepared in $1 \times$ TAE buffer with $15 \mathrm{mM} \mathrm{MgCl}_{2}$. The final DNA origami concentration was set at $10 \mathrm{nM}$. The samples were annealed in a PCR cycler. A DNA anneal program used to fold the DNA MPW was developed by analyzing the origami's melting curve. A qPCR melting curve showed three peaks at $76{ }^{\circ} \mathrm{C}, 70{ }^{\circ} \mathrm{C}$, and $49{ }^{\circ} \mathrm{C}$ which correlated with an increased rate of DNA hybridization. The anneal program is shown in Table S3. The program was designed to hold these three temperatures for relatively long periods. Additionally, to reduce the likelihood of dye degradation, the time spent at elevated temperatures above $77{ }^{\circ} \mathrm{C}$ was minimized. To reduce the effects of photo bleaching and dye degradation all the samples were stored in the dark at $4{ }^{\circ} \mathrm{C}$. Agarose gel electrophoresis was used to evaluate the constructs formation yield. 
Table S3- The anneal program used to fold the six-helix bundle nanotube.

\begin{tabular}{|c|c|c|c|c|}
\hline Step & Temp 1 $\left({ }^{\circ} \mathbf{C}\right)$ & Temp 2 $\left({ }^{\circ} \mathbf{C}\right)$ & ${ }^{\circ} \mathbf{\Delta C} /$ Hour & Time (Min) \\
\hline 1 & 0 & 85 & 0 & 5 \\
\hline 2 & 85 & 77 & -30 & 16 \\
\hline 3 & 77 & 76 & -0.6 & 100 \\
\hline 4 & 76 & 71 & -15 & 20 \\
\hline 5 & 71 & 70 & -0.3 & 200 \\
\hline 6 & 70 & 50 & -15 & 80 \\
\hline 7 & 50 & 49 & -0.3 & 200 \\
\hline 8 & 49 & 4 & -15 & 80 \\
\hline
\end{tabular}

To remove the excess staple strands from the formed origami wire, 50kDa Amicon centrifuge filters were used. The $1 \times \mathrm{TAE}$ with $15 \mathrm{mM} \mathrm{MgCl} \mathrm{m}_{2}$ buffer and samples were cooled in ice bath for approximately 5 to 10 minutes. The sample was cooled down to make the DNA origami wire more robust and less likely to decompose during the purification process. The Amicon filters were rinsed with $1 \times$ TAE with $15 \mathrm{mM}$ $\mathrm{MgCl}_{2}$ prior to applying the origami. During the rinse step the filters were centrifuged at 14,000 rcf for 1 minute. The filters were then inverted and spun again to remove any excess buffer remaining in the bottom of the filter. Next, $380 \mathrm{~mL}$ of chilled $1 \times$ TAE with $15 \mathrm{mM} \mathrm{MgCl}_{2}$ buffer was placed in each Amicon filter along with $120 \mu \mathrm{L}$ of unpurified origami solution. The filters were centrifuged for 3 minutes at 7,000 rcf. The rinse procedure was repeated three times discarding the buffer containing the excess staples and adding $400 \mu \mathrm{L}$ of $1 \times \mathrm{TAE}$ with $15 \mathrm{mM} \mathrm{MgCl} 2$ each time. Gel electrophoresis confirmed that three spin steps was sufficient to remove all access DNA labeled fluorophores from solution. The gel is shown in Figure 2C. After the third centrifugation the Amicon filter containing the purified origami was inverted and placed in a new centrifuge vial and spun at 3,500 rcf for 1 minute to extract the purified origami from the Amicon filter. The purified origami was then diluted with $65 \mu \mathrm{L}$ of $1 \times \mathrm{TAE}$ with $15 \mathrm{mM} \mathrm{MgCl} 2$ to ensure the sample volume and concentration were in the desired ranges. 


\section{AFM}
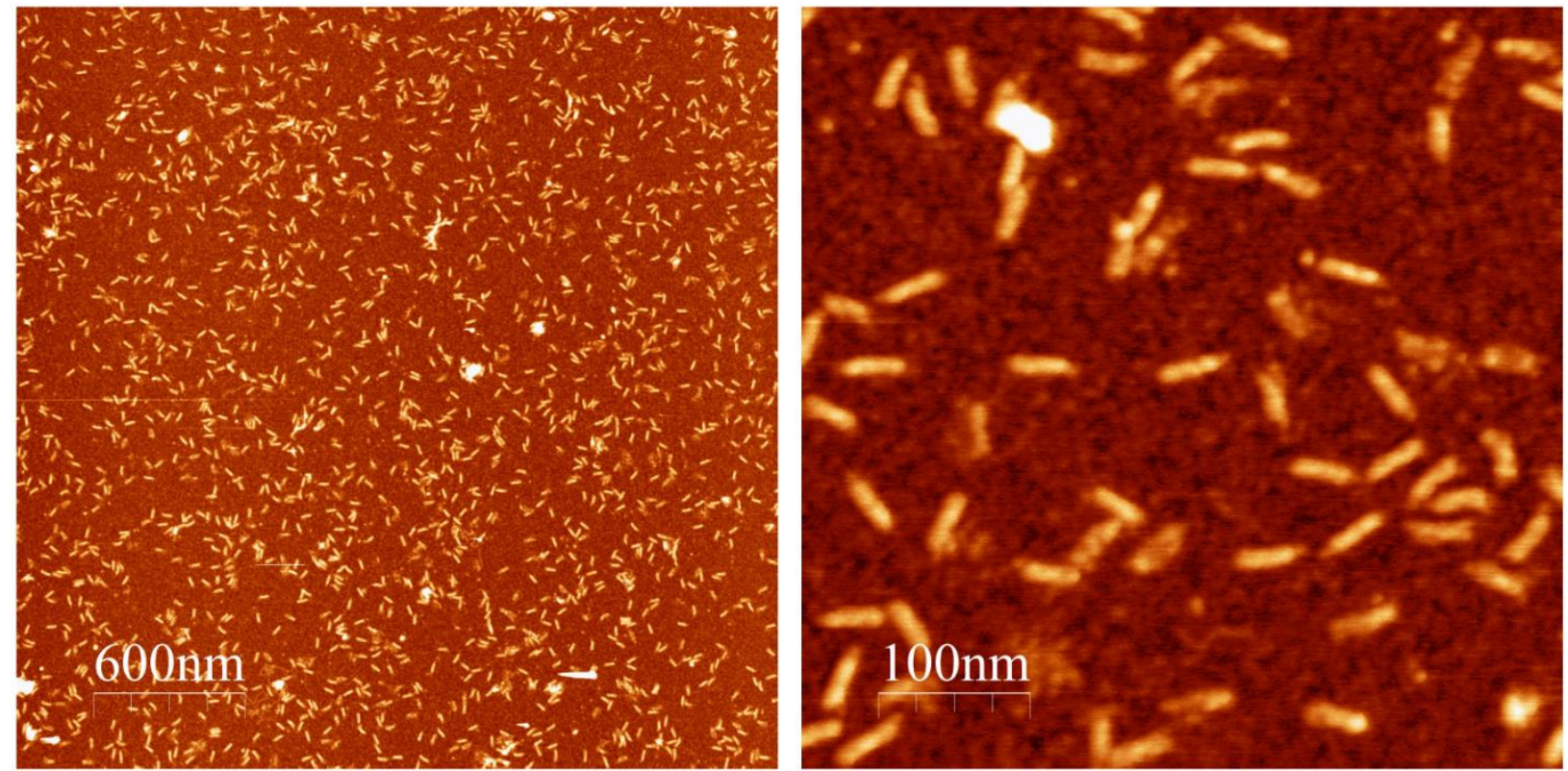

Figure S1: The AFM characterization was performed on Bruker MultiMode 8 AFM with a Nanoscope V controller. Freshly prepared origami wires were first purified in $50 \mathrm{kDa}$ Amicon spin filters to remove excess staple strands. Then $20 \mathrm{uL}$ of the sample was deposited on a freshly cleaved mica disk for 2 minutes. The mica sample was then dried with nitrogen gas followed by a $5 \mathrm{~mL}$ Milli-Q water rinse and then completely dried with nitrogen before imaging.

\section{Fluorescence intensity upon increasing Cy3.5}

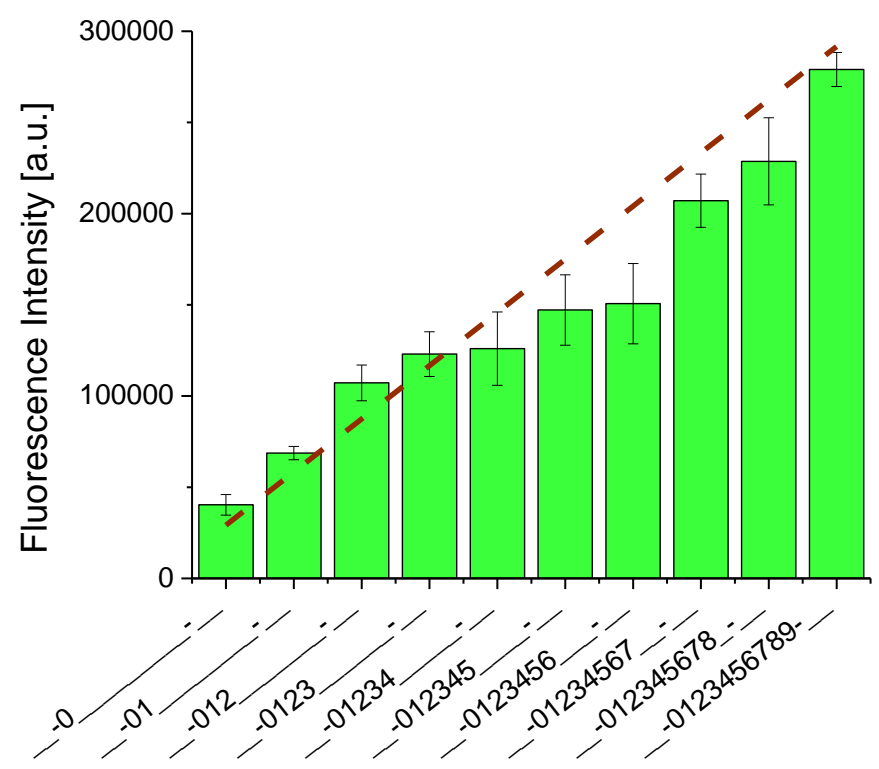

Figure S2: Fluorescence intensity of DNA origami as the HomoFRET relay was built up. Excitation 466 nm. Average intensity 29164 counts per Cy3.5 dye shown in dotted line. 
6. Steady-state determinations of individual FRET efficiencies of the AF488 to Cy3.5 and Cy3.5 to AF647 steps
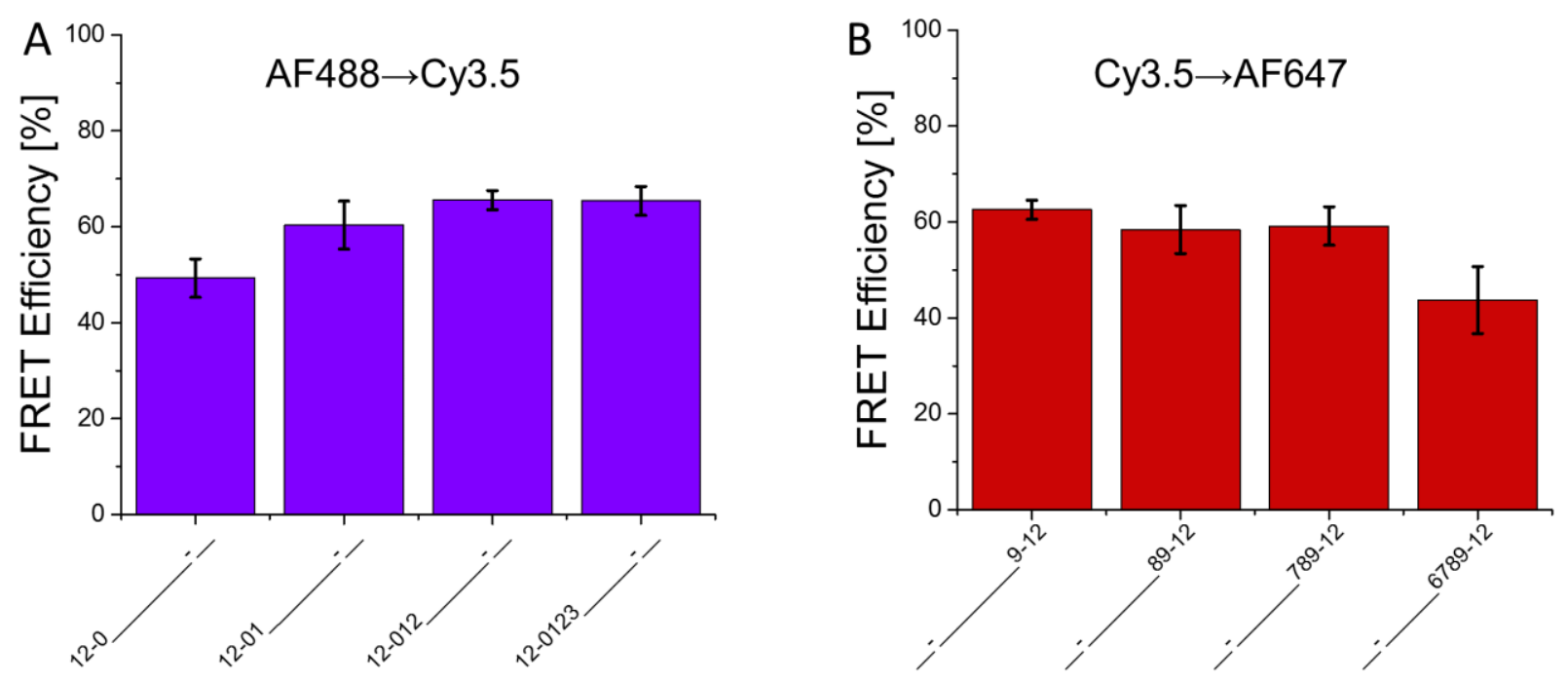

Figure S3: Steady-state FRET efficiency determination based on donor quenching. A) AF488 to Cy3.5 as the number of acceptors increased. B) Cy3.5 to AF647 as the number of donors increased. 


\section{TCSPC fluorescence lifetimes}
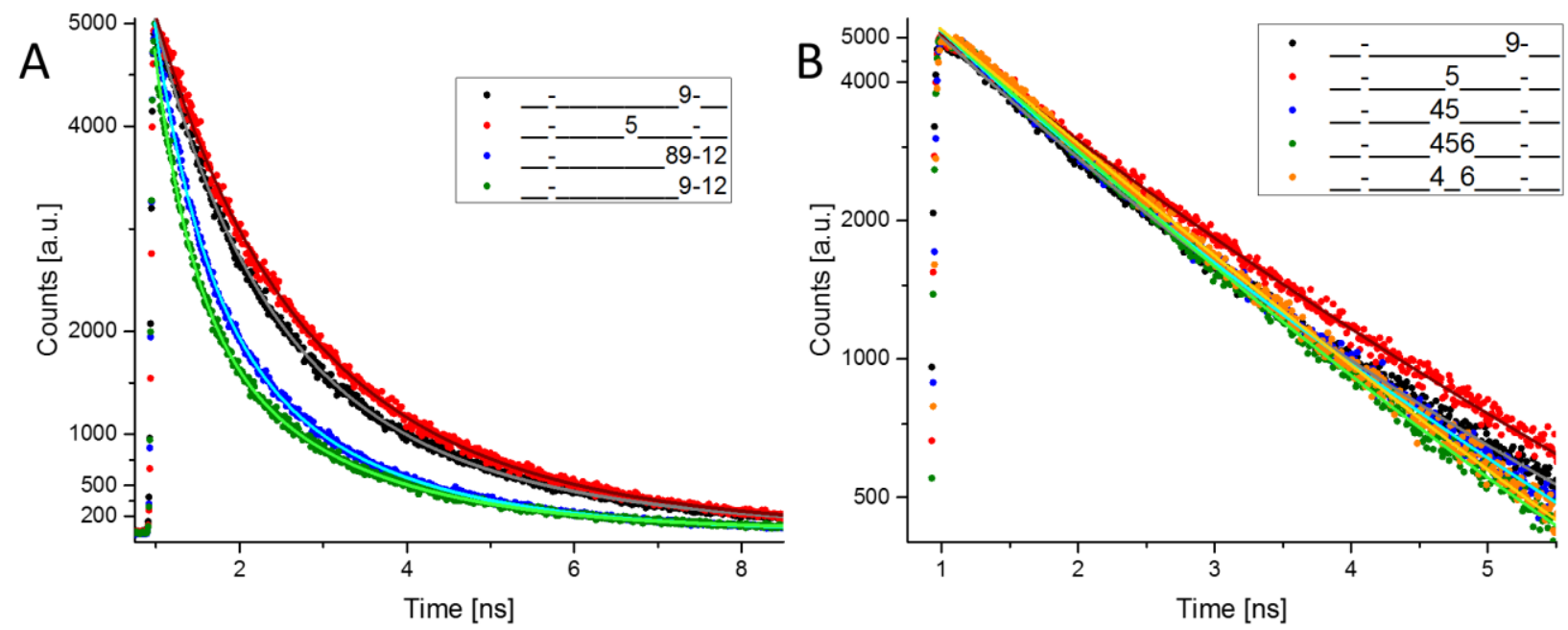

Figure S4: Fluorescence lifetime of DNA MPWs. Fits are dual exponential. We note that the y-axis is in linear (A) and logarithmic (B) scale respectively.

Table S4- Fluorescence lifetime fitting parameters

\begin{tabular}{|c|c|c|c|c|c|}
\hline DNA MPW & $\mathrm{A}_{1}$ & $\tau_{1}$ & $\mathrm{~A}_{2}$ & $\tau_{2}$ & $\langle\tau\rangle$ \\
\hline${ }_{-}^{-} \quad 9-$ & $9752 \pm 655$ & $1.07 \pm 0.06$ & $4289 \pm 766$ & $2.9 \pm 0.4$ & $2.13 \pm 0.27$ \\
\hline- & $23879 \pm 729$ & $0.47 \pm 0.01$ & $4398 \pm 131$ & $1.87 \pm 0.04$ & $1.06 \pm 0.06$ \\
\hline - & $26174 \pm 535$ & $0.54 \pm 0.01$ & $7664 \pm 244$ & $1.87 \pm 0.04$ & $1.21 \pm 0.06$ \\
\hline$--\ldots 5$ & $4590 \pm 751$ & $1.01 \pm 0.14$ & $5183 \pm 898$ & $2.5 \pm 0.2$ & $2.14 \pm 0.33$ \\
\hline --_ 4 -6_-_- & $7750 \pm 719$ & $1.53 \pm 0.09$ & $708 \pm 571$ & $5.4 \pm 0.7$ & $2.48 \pm 0.13$ \\
\hline$--\ldots 45$ & $6900 \pm 417$ & $0.81 \pm 0.07$ & $7350 \pm 661$ & $2.2 \pm 0.1$ & $1.81 \pm 0.18$ \\
\hline-- & $9675 \pm 1037$ & $1.40 \pm 0.09$ & $1461 \pm 1018$ & $3.95 \pm 0.3$ & $2.16 \pm 0.14$ \\
\hline
\end{tabular}

Fit: $y(t)=y_{0}+a_{1} e^{-t / \tau_{1}}+a_{2} e^{-t / \tau_{2}} . \quad\langle\tau\rangle=\left(\left(A_{1} * \tau_{1}^{2}\right)+\left(A_{2} * \tau_{2}^{2}\right)\right) /\left(\tau_{1} A_{1} * \tau_{2} A_{2}\right)$

\section{Lifetime anisotropy fits.}

Table S5- Fluorescence lifetime anisotropy fitting parameters. Data from main text Figure 4B

\begin{tabular}{|l|l|l|l|l|l|l|}
\hline DNA MPW & $\mathrm{y}_{0}$ & $\mathrm{~A}_{1}$ & $\tau_{1}$ & $\mathrm{~A}_{2}$ & $\tau_{2}$ & $\langle\tau\rangle$ \\
\hline-- 5--- & $0.209 \pm 0.001$ & $0.06 \pm 0.01$ & $0.18 \pm 0.06$ & $0.06 \pm 0.01$ & $2.09 \pm 0.04$ & $1.08 \pm 0.09$ \\
\hline--4 4_6_--- & $0.171 \pm 0.002$ & $0.08 \pm 0.01$ & $0.12 \pm 0.04$ & $0.09 \pm 0.02$ & $1.52 \pm 0.18$ & $0.87 \pm 0.10$ \\
\hline$--45---$ & $0.241 \pm 0.002$ & $0.18 \pm 0.03$ & $0.06 \pm 0.03$ & $0.11 \pm 0.02$ & $1.40 \pm 0.15$ & $0.57 \pm 0.04$ \\
\hline$--456---$ & $0.107 \pm 0.001$ & $0.25 \pm 0.04$ & $0.10 \pm 0.02$ & $0.07 \pm 0.02$ & $0.80 \pm 0.16$ & $0.25 \pm 0.02$ \\
\hline
\end{tabular}

Fit: $y(t)=y_{0}+a_{1} e^{-t / \tau_{1}}+a_{2} e^{-t / \tau_{2}} . \quad\langle\tau\rangle=\left(\left(A_{1} * \tau_{1}\right)+\left(A_{2} * \tau_{2}\right)\right) /\left(A_{1} * A_{2}\right)$ 


\section{Additional spectra of MPW in PVA film}
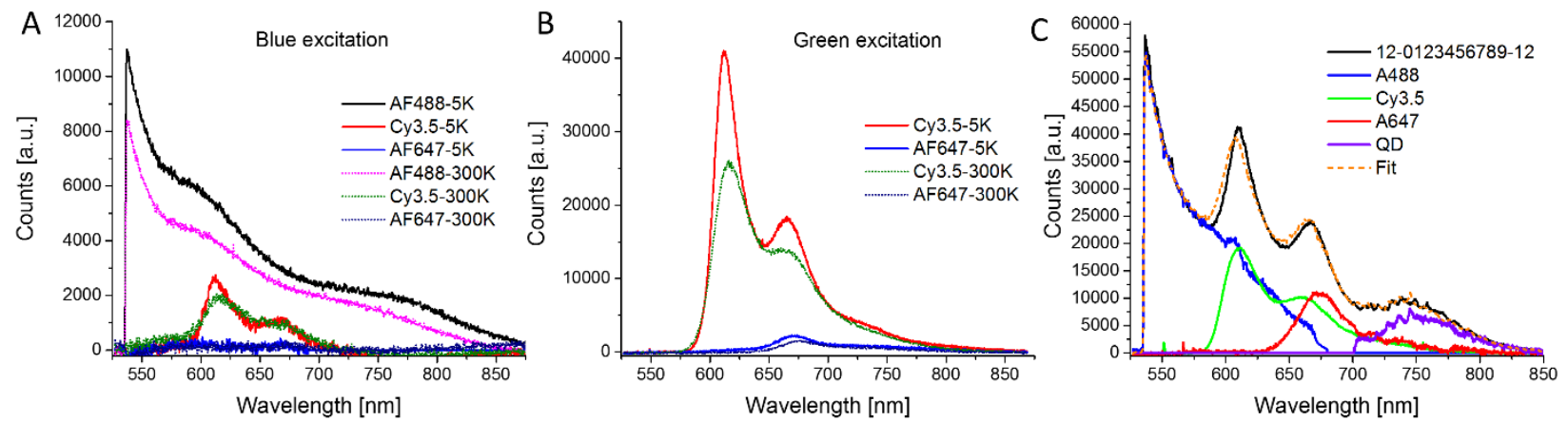

Figure S5: Microscopy fluorescence spectra of individual dye components at 5 or $300 \mathrm{~K}$ excited either with (A) $455 \mathrm{~nm}$ or (B) $532 \mathrm{~nm}$. C) Spectral decomposition of 12-0123456789-12 MPW.
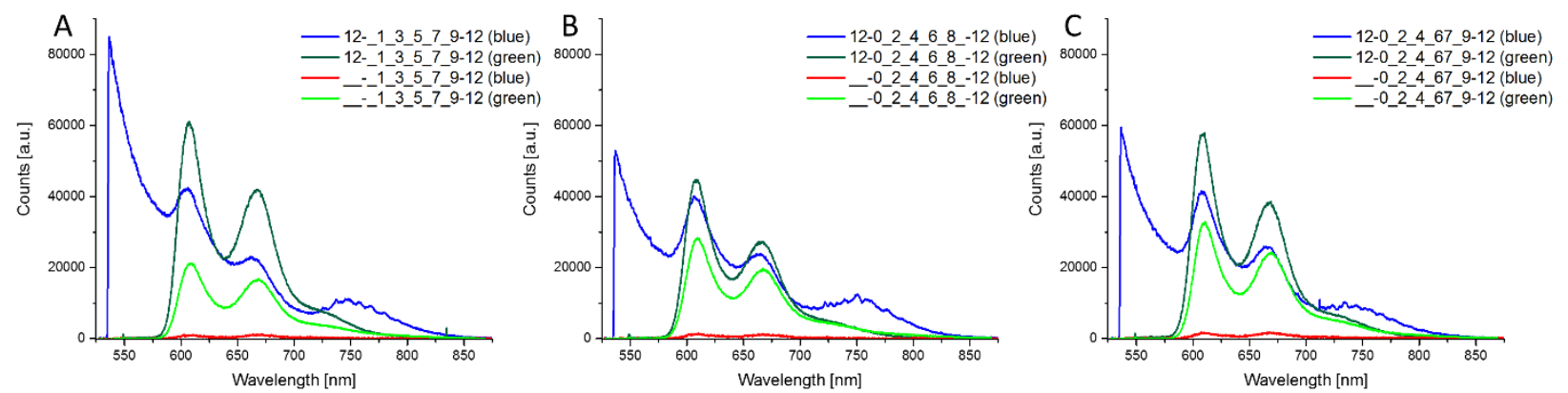

Figure S6: Microscopy fluorescence spectra of representative MPWs along with their corresponding control structures with no AF488. The spectra are at $5 \mathrm{~K}$ and are shown excited with either the blue (455 $\mathrm{nm})$ or green $(532 \mathrm{~nm})$ laser. A) 12-_1_3_5_7_9-12 MPW. B) 12-0_2_4_6_8_-12 MPW. C) 120_2_4_67_9-12 MPW.

Using the spectra of MPWs along with the control structures with no AF488 we can obtain a correction factor, $f$ in equations 1 and 3 (main text), which scales the output considering the downstream excitation. The values for $f$ are 0.53 and 0.61 for measurements at 5 and $300 \mathrm{~K}$ respectively. 


\section{Absorbance Spectra of DNA MPWs}
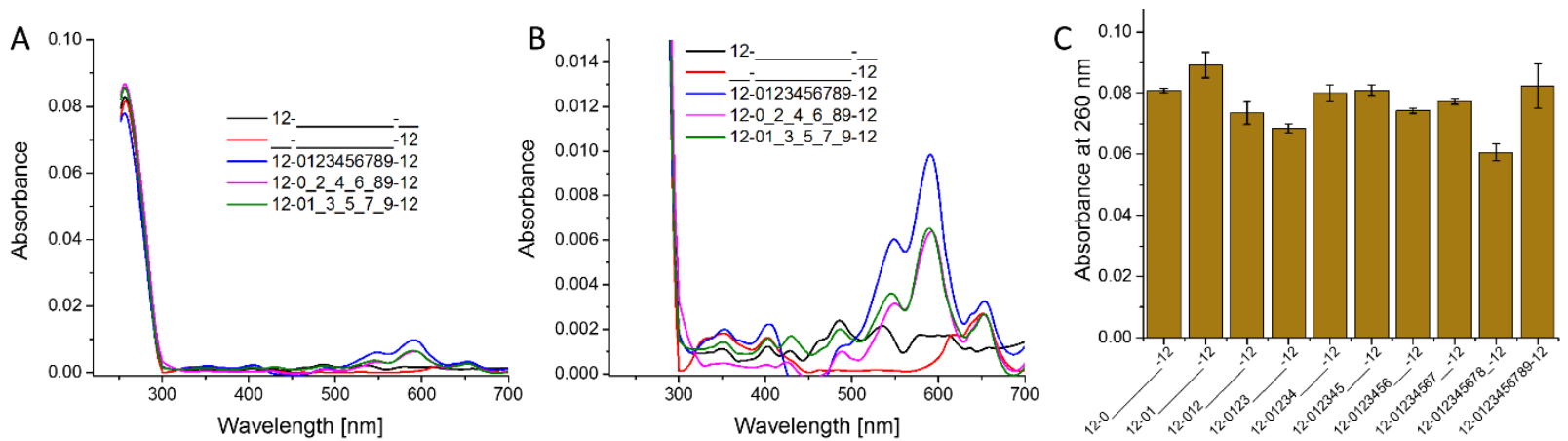

Figure S7: Representative absorbance data. A) Background corrected (scattering) absorbance spectra of DNA MPWs. B) Zoomed in data from A. C) Absorbance spectra at $260 \mathrm{~nm}$ of representative DNA MPWs. Error bars are from 3 separate measurements.

\section{Steady-state fluorescence spectra of DNA MPWs}
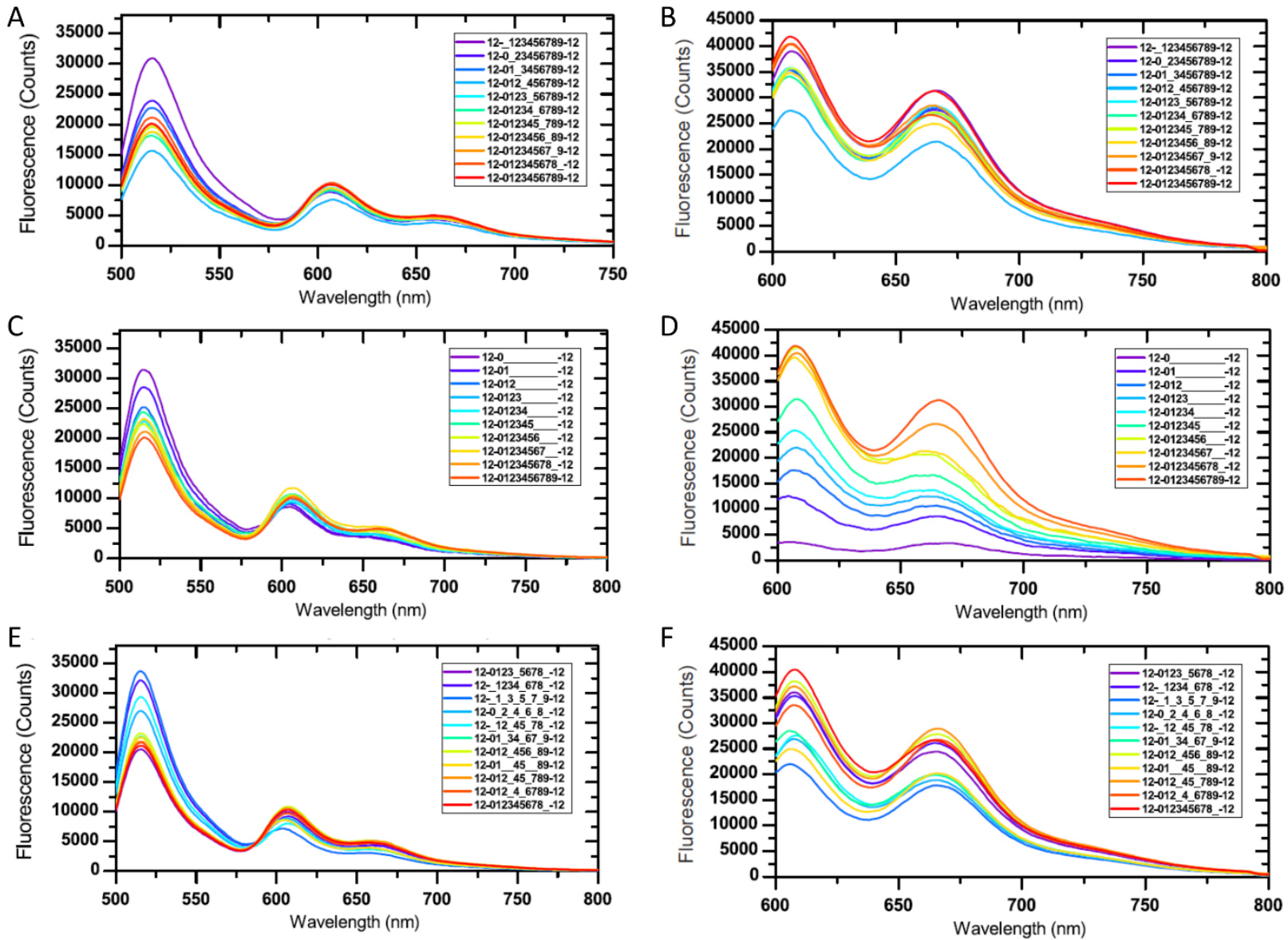

Figure S8: Representative fluorescence spectra of DNA MPWs. A,B) MPW with a single missing Cy3.5. Excited at $466 \mathrm{~nm}$ (A) or $585 \mathrm{~nm}(\mathrm{~B})$. C,D) MPW with increasing number of Cy3.5. Excited at $466 \mathrm{~nm}(\mathrm{C})$ or $585 \mathrm{~nm}$ (D). E,F) Selection of MPW with varying Cy3.5 density. Excited at $466 \mathrm{~nm}$ (E) or $585 \mathrm{~nm}(\mathrm{~F})$ 